\title{
Translation Process Management and Quality Control Based on SDL Trados--A Case Study of Energetic Materials at Extreme Conditions Translation Project
}

\author{
Yahui Wang ${ }^{1, \text { a }}$ \\ ${ }^{1}$ Sinopec Petroleum Engineering Cooperation, Shandong, 257000, China \\ a248182181@qq.com
}

Keywords: Translation process management; quality control; SDL Trados; translation project

\begin{abstract}
This paper presents a modified approach of translation process management and quality control, especially in terminology and corpus management using computer assisted translation software SDL Trados, on Energetic Materials at Extreme Conditions translation project. It highlights the quality of target text and efficiency of translation by calculations of comprehensive error rate and drawing of Gantt Table, in the hope of promoting computer assisted translation and further, established valuable database for future projects.
\end{abstract}

\section{Introduction}

Translation project is one of the myriad temporary endeavors that seek to create a unique product, service, or result, in which case, project management factors, including but not limited to cost, quality, process, manpower, are taken into consideration. Furthermore, project management techniques such like work breakdown structure, Gantt Table, are used to smoothing translation process and quality control. In this case study, the book Energetic Materials at Extreme Conditions, published by Springer, is translated according to academic requirement aiming to introduce advanced technology to Chinese researchers. The source text has total number of 74,456 words and 7 chapters including Figures and tables. The main field of the content discussed in this book is chemical engineering as well as mechanics and mathematics, which suggests that a specialized translation team with professional knowledge of chemistry and linguistic competence is needed.

Translation, by virtue of its multidisciplinary and linguistic transition, is a sophisticated interlingual process, where translators need to have a basic understanding of source language and the disciplines it involves. Nowadays with the help of translation management software based on information and internet technology, knowledge and experience gathered by individual translator during one (even multiple) translation project is shared to all members of the team, thus can be utilized for optimally efficient usage of project, even accessible to other translation projects with similar disciplines and language styles.

This paper studies the translation project based on SDL Trados, a popular computer assisted translation (CAT) software with project management functions, with which translation process and quality control can be easier managed. In this paper, generic project management will be assimilated and modified to specific translation project management that is the subject of the study, meanwhile process management and quality control will be discussed in detail.

\section{Translation Process Management}

\subsection{General Project Management Cycle/Life Cycle}

Project life cycle encompasses a series of stages from project initiation to project closure. Four general stages in the life cycle is summarized by Project Management Body of Knowledge (PMBOK) regardless of project scale and complexity: 1) initiation; 2) organization and preparation; 3) execution; 4) closure (PMBOK, 38). Initiation stage includes but not limited to demand analysis, target, scope, feasibility analysis; organization and preparation stage includes but not limited to schedule planning, human resource management, appointment; execution stage includes but not limited to process control, coordination, modification; closure stage includes but not limited to checking and handover. Each stage, with regard to subsequent breakdown structure, can be further 
divided into these four stages, some of which could be sequentially executed to reduce project uncertainties; others may be done in overlap to conserve execution time.

\subsection{Specified Translation Project Life Cycle}

The life cycle should be modified when it comes to this translation project (Fig. 1). In initiation stage, project manager (PM) analyzed the feasibility of projects available for application, considering overall difficulty, possible time cost, disciplines involved in source text etc. Then PM sent project application, translation application (to Chinese version), and copyright application to Springer, copyright owner of the source text, stating the significance of translation and introduction of this book, and the competence of project team.

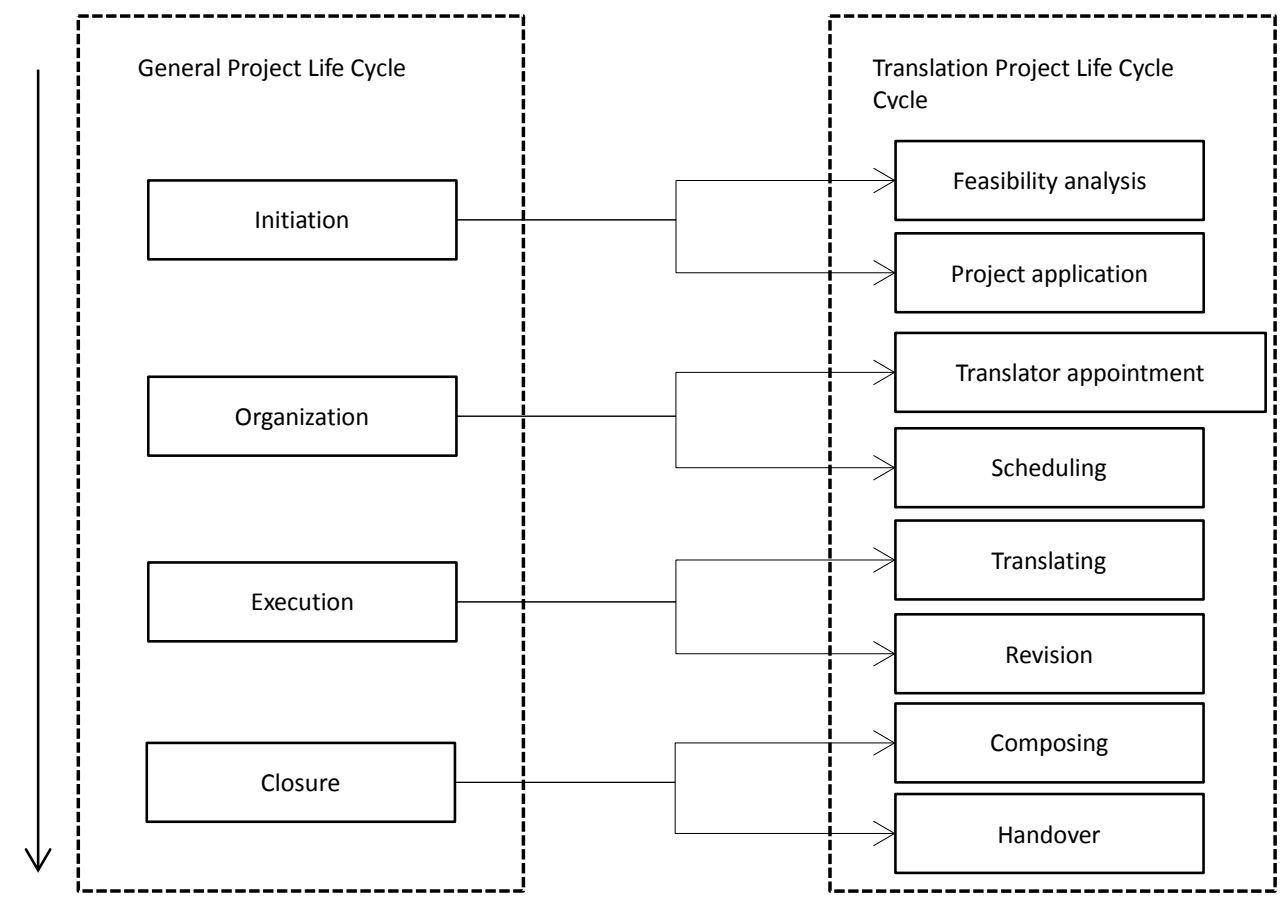

Fig. 1 Modified translation project life cycle

By receiving permission from Springer, project manager signed translation and copyright contract, which is the milestone indicating the end of initiation stage and beginning of organization stage. In this stage, specialized translators who have a working knowledge of chemical engineering, mechanics, and mathematic translation were appointed to this project.

It is significant to point out that by applying CAT software SDL Trados, considerable amount of time was expected to be saved in execution stage---later it turned out that translation and revision time was saved. According to preview of source text, it was recognized that the text is evenly divided into 7 chapters, each of which contains about 10,000 words, and discusses one series of chemical compound. Therefore, 3 translators were appointed, each of who would translate chapter 1 , 2, 3 individually but at the same time, with the help of Trados to establish corpus for further translation. Due to source text familiarizing and corpus establishing, it would take more time in this part of translation. In further parts of translation, however, it is expected that translation time would be apparently conserved because of establishing corpus with Trados.

The significance of Trados is that it can automatically recognize terminologies and translate similar sentences by similarity measure that is by referring to corpus it collects especially terminologies and high frequency phrases and sentences. In the meantime, it will strengthen translation quality of all translators given that all terminologies and language style are integrated and shared. Therefore, it is suggested that corpus accumulation through time would promote translation efficiency, as is described in Fig. 2. Corpus establishing using CAT software SDL Trados will be discussed in detail in the next chapter. 


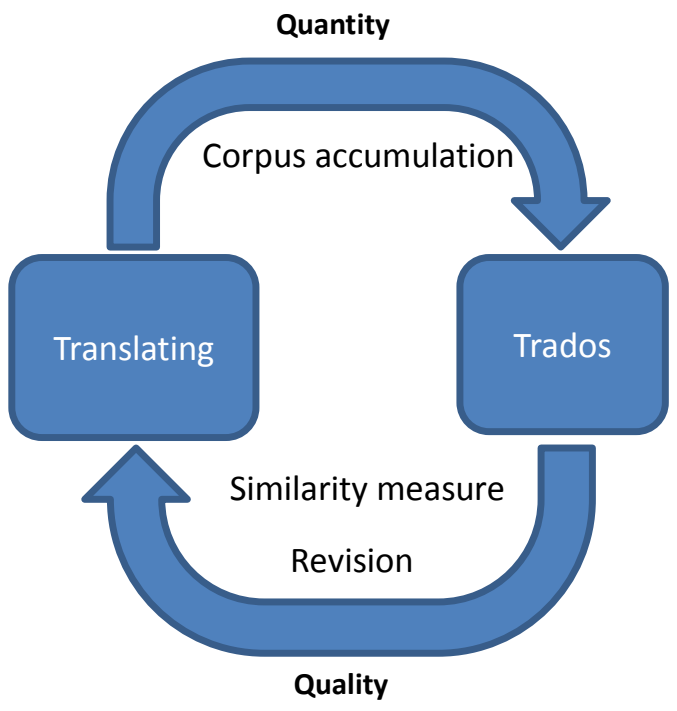

Fig. 2 Relationship between translating quantity and quality.

It is high time to subsequently breakdown the translation process with regard to Trados application. It is also necessary to notice that translation and revision process in this project were done in overlap rather than in linearity. Hence, translation and revision were broke down to 3 parts:

1) Translation of chapter 1,2,3; overlapping with revision;

2) Translation of chapter 5,6; overlapping with revision;

3) Translation of chapter 4,7; overlapping with revision.

This was done for a reason: chapter 1 is the general introduction of the book/source text, which is pivotal of acquiring general knowledge; chapter 2 is methodology, containing mathematical and mechanical guide lines for subsequent chapters; chapter 3 discusses first energetic material of this book, translation of which could serve as a model; chapter 5 and 6 discuss the same series of material; chapter 4 discusses another material and chapter 7 is general conclusion of the book. Together with corpus establishing, the work breakdown structure of translation process can be described as follows:

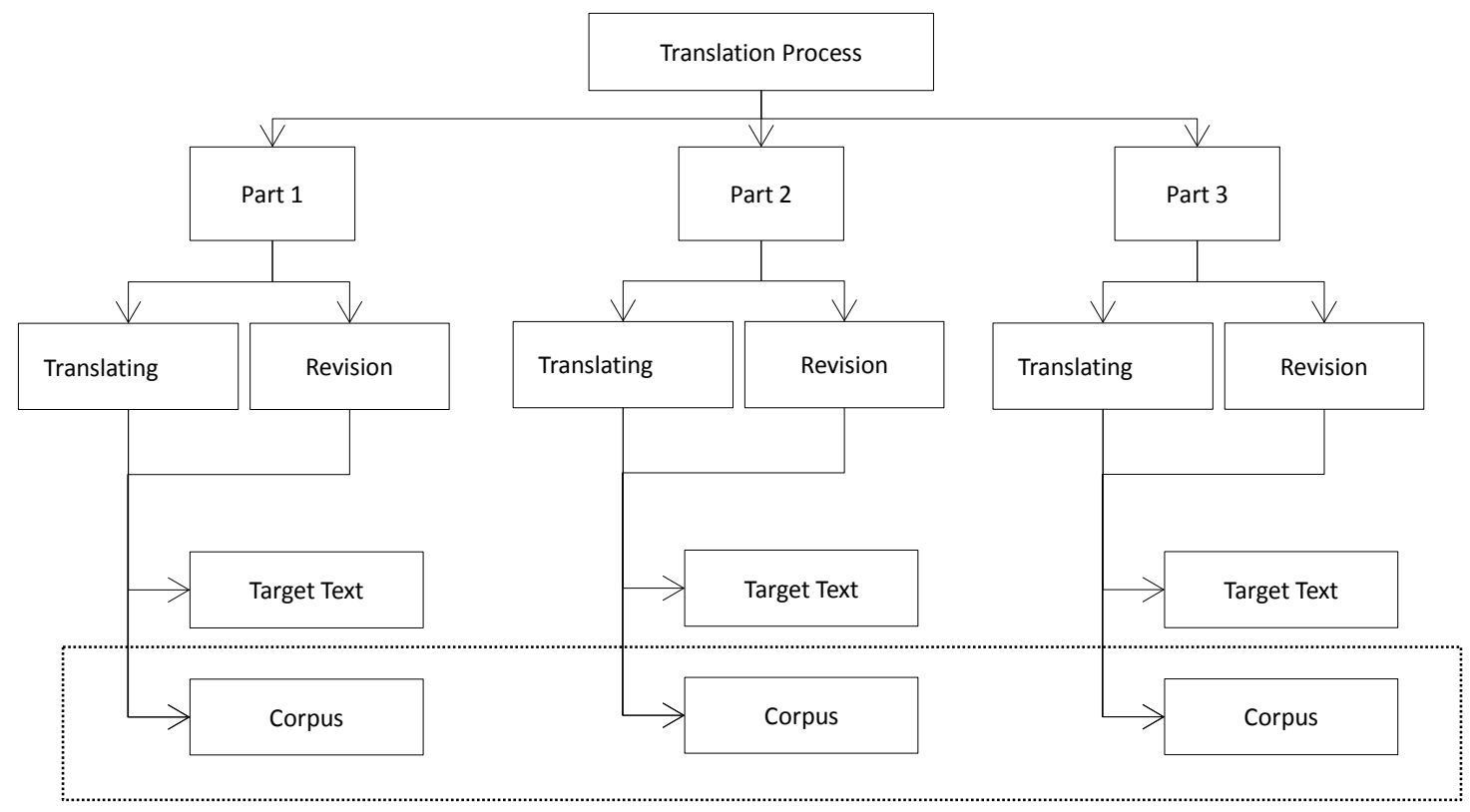

Fig. 3 WBS of translation process.

In each part of process, corpus is collected semi-automatically through translating, and with previous accumulation the translating and revision of other parts would be simplified. 
Throughout the entire translation process, it is summarized that time cost of each part was reduced evidently; meanwhile translation quality has been maintained and promoted incrementally. A Gantt Table presenting overall project schedule and highlighting the modified translation process is then summarized to offer a clear understanding of the project. The Gantt Table is presented as follows:

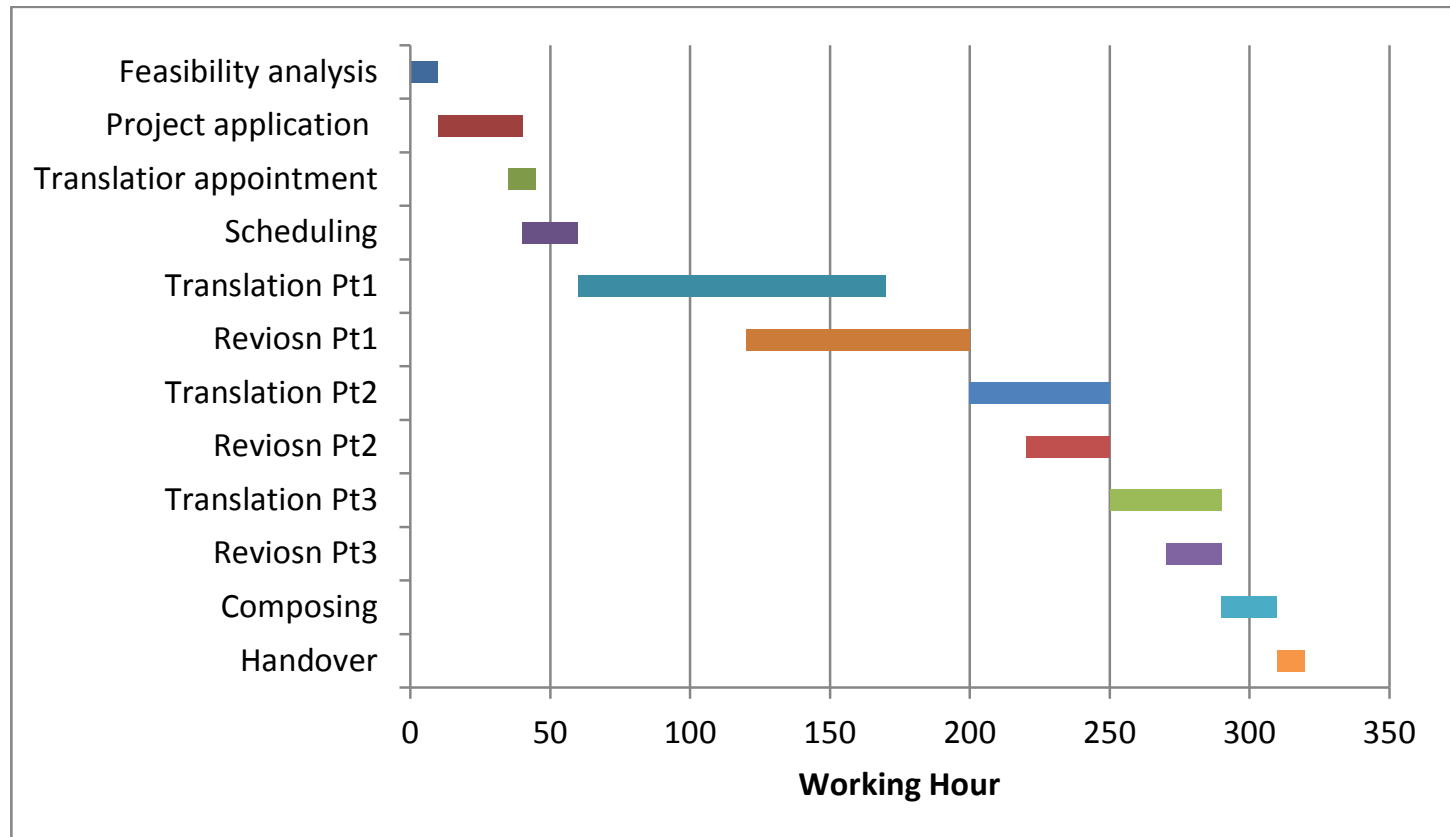

Fig. 4 Gantt Table of Energetic Materials at Extreme Conditions Translation Project

It can be seen many processes overlapping with a certain previous ones, which is considered and eventually proved to save time. The entire time cost is about 320 working hours, which is dramatically less than linear process which would be 430 working hours, regardless of CAT technics employed in translation management. There are 7 milestones respectively located at: the end of feasibility analysis; the end of scheduling; the end of revision of part 1 translation process; the end of revision of part 2 translation process; the end of revision of part 3 translation process; the end of composing; and the end of handover. Each of them marks important point of project timeline with some deliverables (e.g. target text, corpus) to the next stage. It is also apparent that time cost of part 1 translation process is higher than the other two. This is because more time is needed to familiarizing with source text and to establish corpus from the beginning. Furthermore, time consumption is relatively less due to growing familiarity and the assistance of Trados.

\section{Quality control}

In generic term, quality control is "a part of quality management focused on fulfilling quality requirements (ISO 9000)", to identify defects and to maintain quality of products. In translation project, which usually involves multiple translators, translation quality is the main control target during production activity, especially terminology and target language style control. According to human resource management in previous stage, 3 translators appointed are specialized in technical translation, and have working knowledge of chemical, mechanics, mathematics disciplines. Besides, reviewers are chosen from academic researchers who are also masters in these fields. Therefore, it took more time for part 1 translation to integrate terminologies and language styles with cooperation of translator and reviewers, meanwhile corpus has been establishing for future works. In the next two sections, terminology management and similarity measure techniques based on CAT SDL Trados will be introduced to highlight the convenience and efficiency of such tool.

\subsection{Terminology Management and Similarity Measure}

It is essential to integrate translation of all terminologies to their official terms because they always have fixed forms and meanings, and are easy to recognize. Once they are identified and stored in database, they can be shared among all translators and invoked automatically during 
translation. In this project, terminology integration started at the beginning of translation process. Every time a translator identified a terminology, he would report to other translators. Together they discussed and integrated its proper translation, and stored it in the building database. Besides, reviewers who are professional researchers would check whether it is appropriate, if not, he would offer the correct name and modify the database. Moreover, if a term is exotic and the corresponding Chinese term has not been introduced, which occurred a few times during this translation, a meeting would be held with senior professors and specialists to come out the proper name for it. This is why first part of translation took much longer than the other two. As the corpus is establishing, unidentified terminologies became less, easier for subsequent translation, which can be referred to Fig. 2. It is also interesting to point out that terminology database as well as corpus is parts of functions of Trados software. Besides, it can do similarity measure using corpus to promote translation quality and save plenty of time. One sample is presented in Fig. 5.

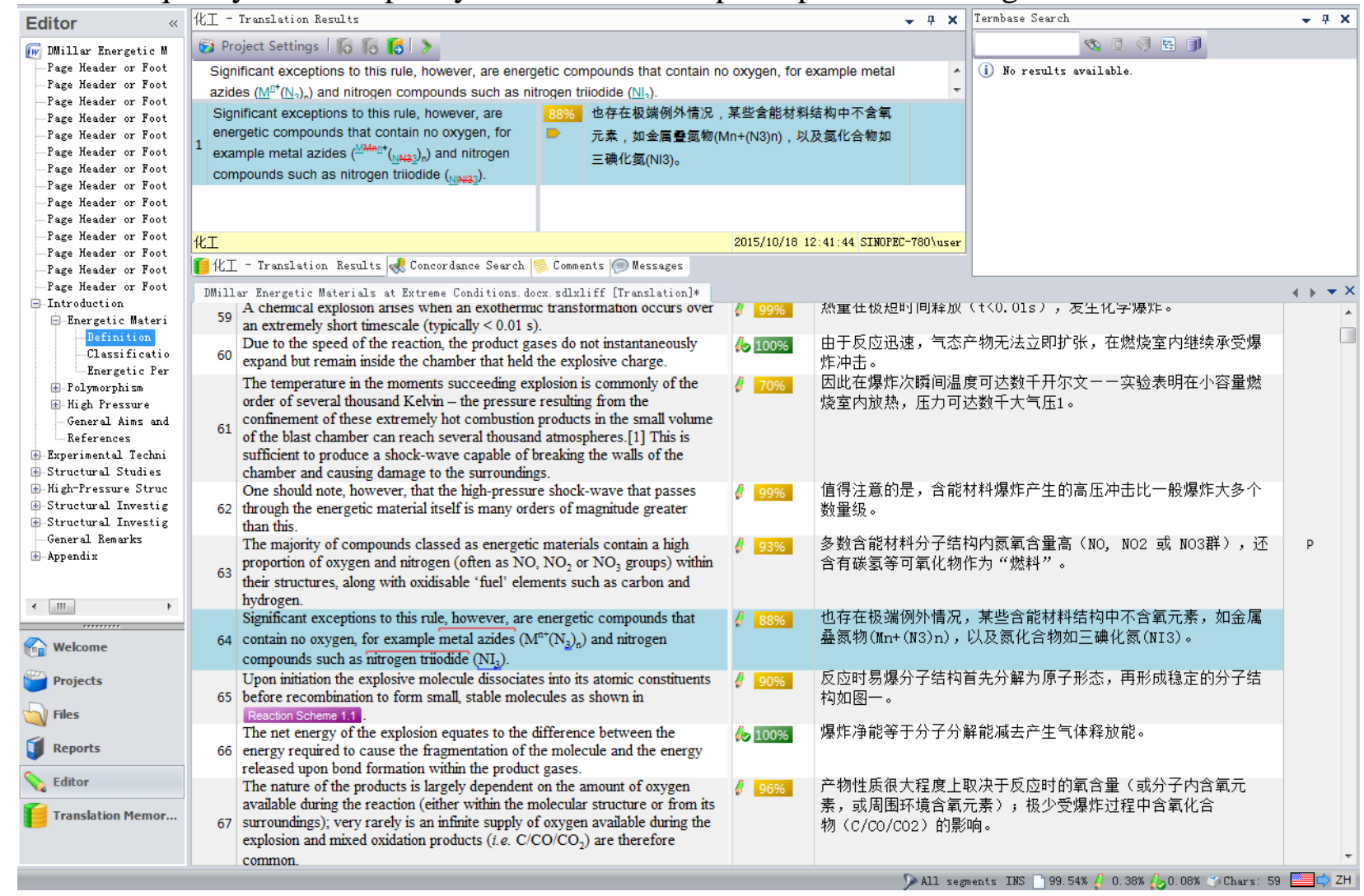

Fig. 5 Sample of similarity measure of terminologies and sentences.

It can be seen from this sample the similarity measure on the right part of the window which are automatically generated in the software by comparison with previous source text and target text. It would be revised by translators later according to percentage of similarity. Furthermore, two terminologies are overlined in the highlighted sentence. This is because these two are automatically recognized and translated by using the database. It is important to note that automatic recognition, including terminology recognition and similarity measure of sentence, is done every time by clicking on each sentence.

\subsection{Statistics of Translation Error Rate}

Quality control techniques are then testified by calculation of translation error rate regulated by Chinese national standard, which is a common method in similar studies [1, 4].

According to GB/T 19682-2005 of China (Target Text Quality Requirements for Translation Services, hereafter referred to as Standard), which is the national standard for translation quality control, the target text which will be used for publication belongs to type I of translation (Standard, section 7.1). Therefore, through comprehensive estimation of type, difficulty, disciplines, language styles, the overall difficulty coefficient value $\mathrm{K}$ is determined as 0.85 . Furthermore, coefficient of target text aim $\mathrm{C}_{\mathrm{A}}$, given its type, is determined as 1. Four types of errors are identified and regulated in the Standard that are: 
Type I: major errors in source text interpretation; major translation errors in keywords, numbers; major missing of translation.

Type II: minor errors in interpretation; translation errors and missing in general words, numbers; ambiguities and grammar errors in interpretation.

Type III: lack of precision, integration, conformance of terminology; or translation errors of terminology.

Type IV: errors in units of measure, symbols, acronyms.

Hence, comprehensive error rate can be calculated by referring to the function presented below:

$$
\text { Comprehensive Error Rate }=\mathrm{K} C_{A} \frac{c_{I} D_{I}+c_{I I} D_{I I}+c_{I I I} D_{I I I}+c_{I V} D_{I V}}{W} \times 100 \%
$$

where $\mathrm{W}$ is total character number; $\mathrm{D}_{\mathrm{I}}, \mathrm{D}_{\mathrm{II}}, \mathrm{D}_{\mathrm{III}}, \mathrm{D}_{\mathrm{IV}}$ are times of occurrence of four types of error; $\mathrm{C}_{\mathrm{I}}, \mathrm{C}_{\mathrm{II}}, \mathrm{C}_{\mathrm{III}}, \mathrm{C}_{\mathrm{IV}}$ are coefficients of four types of error. For detailed information, please refer to Target Text Quality Requirements for Translation Services (GB/T 19682-2005).

Table 1 Statistics of comprehensive error rate of three translation parts

\begin{tabular}{|c|c|c|c|c|c|c|c|}
\hline Item & K & $\mathbf{D}_{\text {I }}$ & $\mathbf{D}_{\text {II }}$ & $\mathbf{D}_{\text {III }}$ & $\mathbf{D}_{\text {IV }}$ & $\mathbf{W}$ & Comprehensive Error Rate \\
\hline Translation Pt1 & 0.85 & 1 & 7 & 311 & 5 & 39,095 & $3.62 \%$ \\
\hline Translation Pt2 & 0.85 & 0 & 4 & 46 & 0 & 31,326 & $0.73 \%$ o \\
\hline Translation Pt3 & 0.85 & 0 & 2 & 1 & 0 & 12,313 & $0.17 \% \mathrm{o}$ \\
\hline
\end{tabular}

This statistics highlights dramatic reduction of number of errors after each part of translation process, giving credits to corpus establishing using Trados. It occurred in part one of translation process a considerable amount of inappropriate terminology translation which was later revised and collected in database. Statistic result meets the requirement of Standard in which it clearly states in Section 7.4 that comprehensive error rate of a quality translation should not surpass $1.5 \%$ per thousand words. The corpus proved to be of great use in identifying and integrating many terminologies reoccurred in subsequent parts, with a few new terminologies ready to be integrated. Besides, language style and reappearing sentences were automatically integrated with the help of Trados. It served as a database and communication bridges to all translators and reviewers of this project, minimizing errors and thus promoting translation quality and saving time.

\section{Conclusion}

Translation projects employing computer assisted translation software would become prevalent because it can give rise to translation quality and save translation and management time if handled properly. The techniques used in this project that are proved viable can offer some enlightenment for future translation project management, as well as the valuable corpus established which if shared later, can be of great help to both individual translator and teams with easy management and high quality.

\section{References}

[1]. Feng Liping. Quality Control through Process Management of Translation Project---A Case Study of the Codex Alimentarius Translation Project [D]. Nanjing: Nanjing Agricultural University, 2013.

[2]. GB/T 19682-2005. Target Text Quality Requirements for Translation Services [S]. Standardization Administration of China (SAC). 2005.

[3]. Project Management Institute. Project Management Body of Knowledge (PMBOK) [M], fifth edition, USA: Project Management Institute, 2008:4-54.

[4]. Pu Xinyue, Gao Jun. Introducing a Translation Project Management Process [J] (in Chinese). Shanghai Journal of Translators, 2014 (2): 35-37.

[5]. SDL. Terminology Survey 2010 [R]. SDL International, 2010. 
[6]. SDL. The Importance of Corporate Terminology [R]. SDL International, 2013.

[7]. Wang Huashu, Zhang Zheng. A Study on Terminology Management in Translation Projects [J] (in Chinese). Shanghai Journal of Translators, 2014 (4): 64-69.

[8]. Yuan Ye, Experimental Report on the Translation Process Management and Quality Control of Three Translation Projects [D]. Nanjing: Nanjing University of Science and Technology, 2014. 